\title{
Mental health sequelae of bone cancer: A narrative review
}

\author{
Christos Tsagkaris 1, Dimitra Desse 2, Emmanouela Dionysia Laskaratou ${ }^{3}$
}

Novel Global Community Educational Foundation, 2270 Hebersham, Australia ${ }^{1}$; Faculty of Medicine, University of Crete, Heraklion, Greece 2 ; University Hospital of Heraklion, Department of Orthopedics, Heraklion, Greece ${ }^{3}$.

\begin{abstract}
Introduction: Bone cancer causes a significant mental health burden. Although the mental health implications of cancer as a whole have been widely discussed, there is limited evidence regarding the psychiatric and psychological sequelae of malignant and benign bone neoplasms in particular.
\end{abstract}

Purpose: To summarize the scientific literature, present relevant knowledge gaps and discuss a number of recommendations. Methodology: The authors searched Pubmed/Medline and Google Scholar with keywords (mental health, bone cancer, musculoskeletal neoplasms, psychiatric comorbidities). When appropriate, MeSH terms and Boolean operators were used. Relevant peer reviewed studies published in English, French or German until 05 May 2021 were included.

Results and Discussion: Loss of mobility, decrease of physical activity, chronic pain and amputations in combination with patient's previous psychiatric history, socioeconomic conditions, cancer biology and treatment-related side effects undermine the mental wellbeing of patients and carers. Since March 2020, the COVID-19 pandemic has posed an additional burden, which is yet to be comprehensively evaluated.

Conclusion: More research and solid action towards the integration of mental health care into the management of bone cancer is crucial.

\section{Keywords}

cancer, bone cancer, osteosarcoma, mental health, depression, anxiety

Address for correspondence:

Christos Tsagkaris, Novel Global Community Educational Foundation, 2270 Hebersham,

Australia.chriss20x@gmail.com

This work is licensed under a Creative Commons Attribution-

NonCommercial 4.0 International License (CC BY-NC 4.0).

(c) (1) (9)

(C) Copyright: Tsagkaris, Desse, Laskaratou, 2021

Submitted for publication: 5 May 2021

Revised: 26 August 2021 Accepted for publication: 16

Licensee NDSAN (MFC- Coordinator of the NDSAN), Italy doi: https://doi.org/10.32437/mhgci.v4i1.131 


\section{Introduction}

Bone malignancies are among the least common types of cancer. In terms of incidence, they have accounted for approximately $0.2 \%$ of all new cancer cases in $2021.0 .3 \%$ of all cancer mortality is associated with bone and joint tumors according to the Surveillance, Epidemiology, and End Results (SEER) Program of the National Cancer Institute of the United States of America ( $\mathrm{NCl}$, 2021). Malignant bone cancers are either primary or secondary. Primary bone tumors, namely chondrosarcoma, osteosarcoma, Ewing's sarcoma, chondrofibrosarcoma, malignant giant cell bone tumor and malignant fibrous histiocytoma, are rarer (Franchi, 2012). Secondary bone tumors usually derive from breast, lung and prostate primary tumors. Bones constitute the third most common metastatic sites (Jayarangaiah , Kemp, Theetha Kariyanna, 2021).

Mental health conditions are quite prevalent among patients with cancer affecting at least $30 \%$ of them. Cancer associated emotional distress, loss of functionality, change of body image, uncertainty, treatment side effects, chronic pain and disease progression contribute to the development of mental health conditions such as anxiety disorder, depression, dementia and substance use among patients with cancer (Niedzwiedz, Knifton, Robb, Katikireddi, Smith, 2019). On top of these, cancer-induced alterations in neurotransmission, hormone secretion and hepatic function can affect patients' conscious and unconscious mental activity (Dai et al., 2020). Although, several aspects of mental health have been investigated in the context of particular types of cancer or neoplastic disease as a whole, to date little is known about mental health and bone cancer.

\section{Purpose}

The purpose of this review is to provide an overview of the available research in the fields, present the knowledge gaps, discuss their implications in contemporary research and clinical practice and outline relevant recommendations.

\section{Results and Discussion}

\section{Aspects of mental health in bone cancer}

During the last years, a limited number of clinical, observational and cross-sectional studies have evaluated the impact of bone cancer on mental wellbeing of patients and patients' cohabitants, the biological underpinnings and certain clinical aspects of bone cancer.

Sachsenmaier and colleagues (2015) assessed the mental health implications of bone sarcoma by means of a structured questionnaire. In a total of 66 eligible participants, $53 \%$ of patients reported an optimistic attitude and 37.5\% reported emotional steadiness. Female patients and patients with less than two offsprings were more prone to pessimistic ideas. Well-educated and married patients were more likely to seek psychological consultation. Elderly patients had a higher likelihood to experience feelings of isolation, while relationship trouble within families was common (Sachsenmaier, Ipach, Kluba, 2015; Storey et al, 2019). In a critical review of evidence published until 2017, Storey and colleagues investigated the effect of bone sarcoma in mental health and quality of life of 8,823 patients reported in a sum of 12 studies. Their analysis indicated that relapse rates was the most common cause of anxiety and depression. Painful tumors were strongly associated with psychiatric comorbidity leading to worse quality of life, higher levels of perceived stress and even marginalization. Amputations were identified as a key factor in mental health deterioration, although a limited number of patients reported self- esteem improvement following amputation procedures (Storey et.al., 2019). The latter was consistent with the findings of Gil et al. (2018), who assessed the mental health implications of amputations by means of a retrospective study involving 46 patients with metastatic osteosarcoma and reported pain remission and improved selfconfidence following amputation (Gil et al, 2019).

The psychobiological aspects of bone cancer have been addressed by $\mathrm{He}$ and colleagues (2019) in an 84-months long clinicolaboratory study investigating the expression of mental health-related proteins in chondrosarcoma tissue samples. Their study showed that death-domainassociated (DAXX) protein, dopamine D3 receptor (DRD3) and disrupted-in-schizophrenia-1 (DISC1) protein were highly expressed in moderately, poorly differentiated and metastatic osteosarcoma tumors. On the contrary, their expression was significantly lower in tissue samples of osteochondroma, a benign tumor. Further analysis associated the presence of these proteins with worse survival rates and outcomes and suggested them as potential chondrosarcoma biomarkers (He et al, 2019).

A recent study by Groot et al. (2021) analyzed the responses of 47 patients - patient cohabitants' pairs to a series of questionnaires with an eye on quality of life, pain, depression, and anxiety. Patients and their cohabitants - who presumably 
acted as informal carers - scored similarly for quality of life. Nevertheless, higher levels of depression and anxiety linked with an overestimation of the patients' burden were detected among the patients' cohabitants (Groot et al, 2021). Finally, Bartels et al. (2021) attempted to map the impact of the COVID-19 pandemic on the emotional status of patients with bone metastases in a sub-study of the PRESENT cohort. Statistical analysis of the responses of 169 patients to a structured questionnaire indicated that $28 \%$ of patients experienced significant levels stress and anxiety associated with the course of the pandemic and the related restrictive measures. $39 \%$ of the patients had similar feelings regardless of the lockdown situation, while $29 \%$ did not mention such feelings. On these grounds the researchers concluded that the pandemic has affected the psychosocial wellbeing of the majority of patients with bone metastases (Bartels, 2021).

\section{Knowledge gaps and the way forward}

It appears that the interest in the mental health implications of bone cancer has increased in the last 5 years. Nevertheless, the available evidence is still scarce and heterogeneous. Most studies agree that bone cancer leads to a quality of life deterioration and undermines patients' and carers' mental wellbeing (Sachsenmaier, Ipach, Kluba, 2015; Storey, 2019; Gil, 2019). Interestingly, it seems that amputations can affect patients' mental wellbeing positively, to the extent that they relieve them of chronic pain and discomfort (Sachsenmaier, Ipach, Kluba, 2015; Storey, 2019). On top of these, biomolecules implicated in mental health disorders have been associated with bone cancer and are further assessed as biomarkers.

Evidently, there is a lack of clinical studies assessing the effectiveness of psychosocial interventions (individual or group oriented psychotherapy and counseling, psychiatric medication, ergotherapy sessions etc) in supporting bone cancer patients' and carers' mental wellbeing (Lingens, Schulz, Bleich; , 2021). During the COVID-19 pandemic digital psychosocial interventions can be implemented and assessed. Telehealth has already been used to perform neuropsychological tests and monitor patients with dementia, who did not have access to physical healthcare facilities due to COVID-19 restrictions (Carotenuto, Traini, Fasanaro, Battineni, Amenta, 2021). Moreover, the impact of amputations and other forms of treatment, such as radiotherapy and chemotherapy, should be assessed in the mental wellbeing of specific vulnerable patients' groups such as children and refugees. Taking into account the cultural factors contributing to pain and disease perception, it is also important to compare patients and carers' attitudes in different continents, countries and social contexts. The aforementioned should also be investigated among healthcare workers. Simultaneously, there is a clear need for more research about the biological interconnection of bone cancer and mental health disorders. Such research can yield biomarkers and potential therapeutic targets with multiple applications in oncology and psychiatry.

Currently, the way forward appears twofold encompassing research and clinical interventions. Research needs to address the knowledge gaps, preferably by means of large-scale studies. Retrospective research appears as the optimal means to assess the potential effects of particular mental health indicators and interventions. Intention - to - treat analysis can be used to evaluate the potential effects of mental health oriented treatment strategies across different study groups. Systematic reviews and metanalysis of the generated evidence can be performed in regular intervals, in order to inform researchers in the field, administrators and policymakers. Clinical interventions can be based on the existing knowledge and psychosocial care practices in cancer. In this context, the concept of a multidisciplinary cancer team including mental health specialists and practitioners should be enhanced (Silbermann, 2013). Healthcare providers and insurance agencies should also take action to provide patients with bone cancer with access to mental health services. Finally, yet importantly, patients' organizations, civil society and health bodies should advocate for the integration of mental health into bone cancer management.

\section{Conclusions (and Future Work)}

Mental health increases the burden of bone cancer. Recent studies have illustrated the psychosocial implications of bone cancer towards patients and carers and its potential biological underpinnings. More research and solid action towards the integration of mental health care into the management of bone cancer is crucial.

\section{Conflict of interest}

The author declares that she has no conflict of interests.

\section{References}


Cancer of the bones and joints - cancer stat facts. National Cancer Institute; Surveillance, Epidemiology and End Results Program. (n.d.). Retrieved October 1, 2021, from https://seer.cancer.gov/statfacts/htm//bones.ht $\underline{\mathrm{ml}}$

Franchi A. (2012). Epidemiology and classification of bone tumors. Clinical cases in mineral and bone metabolism : the official journal of the Italian Society of Osteoporosis, Mineral Metabolism, and Skeletal Diseases, 9(2), 92-95. Jayarangaiah A, Kemp AK, Theetha Kariyanna P. Bone Metastasis. [Updated 2021 Aug 7]. In: StatPearls [Internet]. Treasure Island (FL): StatPearls Publishing; 2021 Jan-. Available from: https://www.ncbi.nlm.nih.gov/books/NBK50791 1/

Niedzwiedz, C. L., Knifton, L., Robb, K. A., Katikireddi, S. V., \& Smith, D. J. (2019). Depression and anxiety among people living with and beyond cancer: a growing clinical and research priority. BMC cancer, 19(1), 943. https://doi.org/10.1 186/s12885-019-6181-4

Dai, S., Mo, Y., Wang, Y., Xiang, B., Liao, Q., Zhou, M., Li, X., Li, Y., Xiong, W., Li, G., Guo, C., \& Zeng, Z. (2020). Chronic Stress Promotes Cancer Development. Frontiers in oncology, 10, 1492. https://doi.org/10.3389/fonc. 2020.01492

Sachsenmaier, S. M., Ipach, I., \& Kluba, T. (2015). Quality of Life, Physical and Mental Status and Contentment of Patients with Localized Soft Tissue or Bone Sarcoma: A Questionnaire Analysis. Orthopedic reviews, 7(2), 5920. https://doi.org/10.4081/or.2015.5920

Storey, L., Fern, L. A., Martins, A., Wells, M., Bennister, L., Gerrand, C., Onasanya, M., Whelan, J. S., Windsor, R., Woodford, J., \& Taylor, R. M. (2019). A Critical Review of the Impact of Sarcoma on Psychosocial Wellbeing. Sarcoma, 2019, 9730867. https://doi.org/10.1155/2019/9730867

Gil, S., Fernandez-Pineda, I., Rao, B., Neel, M. D., Baker, J. N., Wu, H., Wu, J., \& Anghelescu, D. L. (2019). Role of Amputation in Improving Mobility, Pain Outcomes, and Emotional and Psychological Well-Being in Children With Metastatic Osteosarcoma. The American journal of hospice \& palliative care, 36(2), 105110.

https://doi.org/10.1177/1049909118791119

He, L., Shi, X., Chen, R., Wu, Z., Yang, Z., \& Li, Z. (2019). Association of Mental Health-Related
Proteins DAXX, DRD3, and DISC1 With the Progression and Prognosis of Chondrosarcoma. Frontiers in molecular biosciences, 6, 134. https://doi.org/10.3389/fmolb.2019.00134

Groot, O. Q., Paulino Pereira, N. R., Bongers, M., Ogink, P. T., Newman, E. T., Verlaan, J. J., Raskin, K. A., Lozano-Calderon, S. A., \& Schwab, J. H. (2021). Do Cohabitants Reliably Complete Questionnaires for Patients in a Terminal Cancer Stage when Assessing Quality of Life, Pain, Depression, and Anxiety?. Clinical orthopaedics and related research, 479(4), 792-801.

https://doi.org/10.1097/CORR.0000000000001 525

Bartels, M., Gal, R., van der Velden, J. M., Verhoeff, J., Verlaan, J. J., \& Verkooijen, H. M. (2021). Impact of the COVID-19 pandemic on quality of life and emotional wellbeing in patients with bone metastases treated with radiotherapy: a prospective cohort study. Clinical \& experimental metastasis, 38(2), 209-217. https://doi.org/10.1007/s10585-021-10079-x

Lingens, S. P., Schulz, H., \& Bleich, C. (2021). Evaluations of psychosocial cancer support services: A scoping review. PloS one, 16(5), e0251126.

https://doi.org/10.1371/journal.pone.0251 126

Carotenuto, A., Traini, E., Fasanaro, A. M., Battineni, G., \& Amenta, F. (2021). TeleNeuropsychological Assessment of Alzheimer's Disease. Journal of personalized medicine, $11(8)$, 688. https://doi.org/10.3390/jpm1 1080688

Leontjevas, R., Knippenberg, I., Bakker, C., Koopmans, R., \& Gerritsen, D. L. (2021). Telehealth and telecommunication in nursing homes during COVID-19 antiepidemic measures in the Netherlands. International

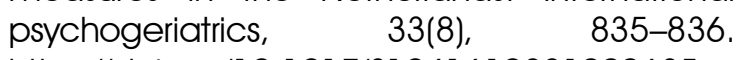
https://doi.org/10.1017/S1041610221000685

Sillbermann, M., Pitsillides, B., Al-Alfi, N., Omran, S., Al-Jabri, K., Elshamy, K., Ghrayeb, I., Livneh, J., Daher, M., Charalambous, H., Jafferri, A., Fink, R., \& El-Shamy, M. (2013). Multidisciplinary care team for cancer patients and its implementation in several Middle Eastern countries. Annals of oncology : official journal of the European Society for Medical Oncology, 24 Suppl 7(Suppl 7), vii41-vii47. https://doi.org/10.1093/annonc/mdt265 